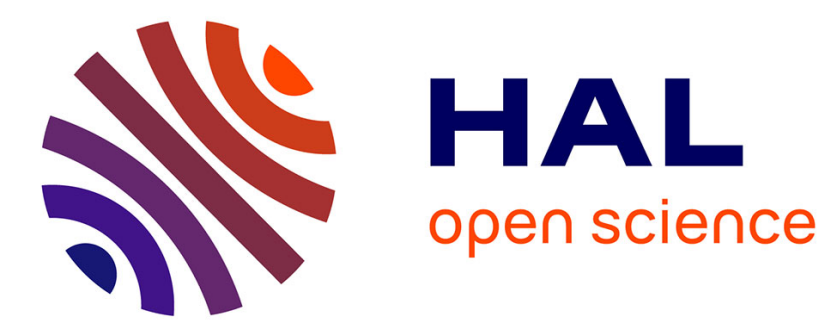

\title{
Complementary Observer for Body Segments Motion Capturing by Inertial and Magnetic Sensors
}

\author{
Hassen Fourati, Noureddine Manamanni, Lissan Afilal, Yves Handrich
}

\section{To cite this version:}

Hassen Fourati, Noureddine Manamanni, Lissan Afilal, Yves Handrich. Complementary Observer for Body Segments Motion Capturing by Inertial and Magnetic Sensors. IEEE/ASME Transactions on Mechatronics, 2014, 19 (1), pp.149-157. 10.1109/TMECH.2012.2225151 . hal-00690145v2

\section{HAL Id: hal-00690145 \\ https://hal.science/hal-00690145v2}

Submitted on 1 Feb 2013

HAL is a multi-disciplinary open access archive for the deposit and dissemination of scientific research documents, whether they are published or not. The documents may come from teaching and research institutions in France or abroad, or from public or private research centers.
L'archive ouverte pluridisciplinaire HAL, est destinée au dépôt et à la diffusion de documents scientifiques de niveau recherche, publiés ou non, émanant des établissements d'enseignement et de recherche français ou étrangers, des laboratoires publics ou privés. 


\title{
Complementary Observer for Body Segments Motion Capturing by Inertial and Magnetic Sensors
}

\author{
Hassen Fourati, Noureddine Manamanni, Member, IEEE, Lissan Afilal, and Yves Handrich
}

\begin{abstract}
This paper presents a viable quaternion-based complementary observer (CO) that is designed for rigid body attitude estimation. We claim that this approach is an alternative one to overcome the limitations of the extended Kalman filter. The CO processes data from a small inertial/magnetic sensor module containing triaxial angular rate sensors, accelerometers, and magnetometers, without resorting to GPS data. The proposed algorithm incorporates a motion kinematic model and adopts a two-layer filter architecture. In the latter, the Levenberg Marquardt algorithm preprocesses acceleration and local magnetic field measurements, to produce what will be called the system's output. The system's output together with the angular rate measurements will become measurement signals for the $\mathrm{CO}$. In this way, the overall $\mathrm{CO}$ design is greatly simplified. The efficiency of the $\mathrm{CO}$ is experimentally investigated through an industrial robot and a commercial IMU during human segment motion exercises. These results are promising for human motion applications, in particular future ambulatory monitoring.
\end{abstract}

Index Terms-Complementary observer (CO), inertial measurement unit (IMU), motion capture, quaternion, wearable MEMS sensors.

\section{INTRODUCTION}

M OTION tracking is the key technology in many fields such as stroke rehabilitation [1], gait analysis [2], human motion monitoring [3], and other applications that require continuous information about body attitude. The attitude acts as a tool for biologists to enable them to estimate the power transfer between the human body and the environment [4] in many applications such as stride analysis, physical labor, and rehabilitation [5]. A number of motion tracking systems have already been developed for human motion including mechanical, magnetic, optical, acoustic, and inertial/magnetic tracking systems [1].

Manuscript received March 14, 2012; revised June 12, 2012 and September 7, 2012; accepted October 7, 2012. Recommended by Technical Editor W.-J. Kim. This work was supported by the Alsace and Champagne-Ardenne Regions within the framework of the project "NaviMeles."

H. Fourati is with the GIPSA-Lab, NeCS Team, Department of Automatic Control, UMR 5216 CNRS, University of Joseph Fourier, Grenoble, France (e-mail: hassen.fourati@ gipsa-lab.grenoble-inp.fr).

N. Manamanni and L. Afilal are with the Automatic Control Group, CReSTIC-URCA, University of Reims Champagne-Ardenne, 51100 Reims, France (e-mail: noureddine.manamanni@univ-reims.fr; lissan.afilal@ univ-reims.fr).

Y. Handrich is with IPHC-DEPE-CNRS, UMR 7178, Strasbourg University, 67087 Strasbourg, France (e-mail: yves-jean.handrich@iphc.cnrs.fr).

Color versions of one or more of the figures in this paper are available online at http://ieeexplore.ieee.org.

Digital Object Identifier 10.1109/TMECH.2012.2225151
Mechanical tracking systems use an exoskeleton that is attached to the articulated structure intended to be tracked [6]. Goniometers within the skeletal linkages measure joint angles. These systems only track one single rigid body. Optical tracking systems using markers and vision are recently reported in the literature. Image-based systems determine position using multiple cameras to track predesignated points on moving objects within a working volume [7]. However, the use of markers is limited to small areas and the approach using these tools needs specific material (markers and cameras), which is relatively expensive or not user friendly.

Body tracking using inertial and magnetic sensors has recently attracted a strong interest. Inertial and magnetic tracking systems use a combination of inertial sensors (a set of accelerometers and gyroscopes) and magnetic sensors (magnetometer) [8]. There is no inherent latency associated with this sensing technology and all delays are due to data transmission and processing. Another benefit is its lack of necessary source, whereas electromagnetic, acoustic, and optic devices require emissions from a source to track objects. Due to the recent technological advances, inertial and magnetic sensors have become available with low-cost, small-size, and low-energy consumption [9]. This helped to build wrist-watch-sized, self-contained inertial/magnetic sensor modules. Consequently, human motion estimation can be tracked outdoor using smaller ambulatory measurement systems.

Inertial and magnetic sensors have varying advantages and drawbacks. Accelerometers theoretically measure the sum of linear acceleration and gravity [9]. In quasi-static situations, the linear acceleration can be neglected with respect to the gravity [10]. Measuring the gravity in the sensor coordinate frame using accelerometers allows the estimation of orientation relative to the horizontal plane. However, in a dynamic situation (free motion) the accelerometer measures linear acceleration and gravity. In this case, it is not easy to dissociate these two physical quantities, and thus, it becomes difficult to calculate the attitude accurately. Gyroscopes measure angular velocity and estimate a change in orientation. However, this solution would be prone to drifting over time due to the build-up of bias and drift errors [11]. Magnetometers are used to measure the local magnetic field vector in sensor coordinates and allow the determination of orientation relative to the vertical axis, which provides additional information regarding orientation [12]. The main problem with magnetometers is the influence of ferrous material. An in-depth analysis in [13] describes some experiments carried out to examine and characterize small-scale magnetic interference caused by typical objects and how this interference affects the accuracy of orientation estimates from 
inertial/magnetic sensor modules. The only recommendation stated in [13] is to maintain a distance of approximately $1 \mathrm{~m}$ from the source of interference. Since each sensor shows some advantages and drawbacks, the key element of the study is how to combine these three data in order to improve the quality of the motion reconstruction.

Many studies of motion tracking using inertial and magnetic sensors have been performed using a number of fusion techniques in order to overcome the drawbacks that appear when we use each sensor individually. The orientation drift resulting from gyroscope bias [11] can be bounded by additional sensors. In [2] and [14], the authors describe an attitude estimation algorithm based on a three-axis gyroscope and three-axis accelerometer data. The proposed approaches used the cyclical nature of human gait and a Kalman filter (KF). The change in orientation calculated using gyroscopes is fused with the inclination measured by accelerometers to estimate an accurate orientation even with the presence of accelerations. In [15], an orientation tracking system is performed using nine-axis sensor modules containing three orthogonally mounted triads of angular rate sensors, accelerometers, and magnetometers. A linear $\mathrm{KF}$ was designed to process the sensor signals and to estimate the desired sensing variables of the gravity and magnetic field and further the orientation of the body segment. This nineaxis sensor module is also used in [16] to develop an extended Kalman filter (EKF) for real-time estimation of human limb segment orientation. This EKF presents some drawbacks such as the difficulty to guarantee the global convergence of the filter due to the linear approximation of its nonlinear process model. The work reported in [17] considered the subproblem of using integrated inertial and magnetic sensing to track the attitude. Experiments are performed to validate the developed KF only using small clinical motion and a small rotational perturbation. A wearable sensing system is developed in [18] for the tracking and monitoring of functional arm movement. A body-sensing module was designed, integrating an optical linear encoder and an accelerometer. The authors concluded that the best reconstructed human motion is still not perfect. Recently, the authors in [19] have described a method to determine the attitude of a human body based on micro inertial/magnetic sensor units. The approach employs a KF. A commercial inertial measurement unit (IMU) is used in [20] to measure the attitude, necessary for biofeedback problems.

Through the cited data fusion approaches, a multitude of attitude representation is used. Quaternion is introduced to improve computational efficiency and to avoid singularities. Due to the unconventional nature of quaternion kinematics, the filtering approaches have been designed in two different ways according to the formulation of the error vector and the update of state estimates. We can differentiate between the additive and multiplicative quaternion techniques [5], [16], [21], [22]. These two techniques are used in the calculation of error vector and in the update of state estimates. The developed EKF in [16] is based on a quaternion additive technique. This approach is easy to implement but is considered as localized approximation since it is valid for only small attitude changes. A complementary filter is developed in [5], [22] based on quaternion multiplicative technique and can be applied for larger attitude maneuvers. It will also be used during this study.

In this paper, a complementary observer (CO) approach is developed for human segment motion estimation. In order to produce 3-D orientation estimates relative to an Earth-fixed reference frame, the $\mathrm{CO}$ uses input from a sensor module containing a triad of orthogonally mounted accelerometers, angular rate sensors, and magnetometers. Quaternion is used to represent the attitude. The algorithm incorporates a motion kinematic equation to model the human segment motion. It adopts a two-layer filter architecture in which the Levenberg Marquardt algorithm (LMA) preprocesses acceleration and local magnetic field measurements to produce what will be referred to as the system's output. The latter together with the angular rate data becomes measurements for the $\mathrm{CO}$. In this way, the overall $\mathrm{CO}$ design is greatly simplified.

The primary contributions of this paper can be stated within the following points.

1) The experimental validation of the $\mathrm{CO}$ performance for human posture tracking applications.

2) The development of estimation approach without the intermediate step of gyro bias estimation/correction like previous attitude estimation approaches [21], [23].

3) The data fusion of inertial and magnetic measurements without resorting to GPS data.

4) The use of robust observer instead of EKF. EKF presents some drawbacks such as the difficulty in guaranteeing the global convergence of the filter due to the linear approximation of the nonlinear process model [24].

5) The development of an observer based on a quaternion multiplicative technique, rarely used in the literature although it is more convenient for the nature of quaternion algebra.

This paper is organized as follows. Section II details the materials used and methods: the problem position, the physical system, and the proposed CO for attitude estimation. Section III explains the experimental methodology deployed on a robot and a human subject. Section IV is devoted to the results interpretation and discussion. The final section provides some conclusions and future work.

\section{Materials AND MethodS}

\section{A. Physical System and Process Model}

As stated previously, the objective of this paper is to design a CO for body motion estimation. Therefore, it is necessary to establish a process model representing body motion dynamics. We adopted the attitude kinematic differential equation (1) used in [25] to describe the motion of a rigid body

$$
\dot{q}_{r}=\frac{1}{2} q_{r} \otimes \bar{\omega}_{g}
$$

where $q_{r}=\left[\begin{array}{ll}q_{r, 0} & q_{r, \text { vect }}^{T}\end{array}\right]^{T}$ is the unit quaternion that denotes the mathematical representation of a rigid body attitude between two frames: 1$)$ body-fixed frame $B\left(X_{B}, Y_{B}, Z_{B}\right)$ and 2) earth-fixed frame $N\left(X_{N}, Y_{N}, Z_{N}\right)$. The $X_{N}$-axis points true north. The $Z_{N}$-axis points toward the interior of the Earth, 
perpendicular to the reference ellipsoid. The $Y_{N}$-axis completes the right-handed coordinate system, pointing east (NED: north, east, down).

The unit quaternion $q_{r}$ is expressed as

$$
q_{r}=q_{r, 0}+q_{r, \text { vect }}=q_{r, 0}+q_{r, 1} i+q_{r, 2} j+q_{r, 3} k
$$

where $\quad q_{r, \text { vect }}=q_{r, 1} i+q_{r, 2} j+q_{r, 3} k=\left[\begin{array}{lll}q_{r, 1} & q_{r, 2} & q_{r, 3}\end{array}\right]^{T}$ represents the imaginary vector and $q_{r, 0}$ is the scalar element.

The quaternion product of $q_{r a}=\left[\begin{array}{ll}q_{r a 0} & q_{r a, \text { vect }}^{T}\end{array}\right]^{T}$ and $q_{r b}=\left[\begin{array}{ll}q_{r b 0} & q_{r b, \text { vect }}^{T}\end{array}\right]^{T}$ is defined as

$$
q_{r a} \otimes q_{r b}=\left[\begin{array}{cc}
q_{r a 0} & -q_{r a, \text { vect }}^{T} \\
q_{r a, \text { vect }} & I_{3 \times 3} q_{r a 0}+\left[q_{r a, \text { vect }}^{\times}\right.
\end{array}\right]\left[\begin{array}{c}
q_{r b 0} \\
q_{r b, \text { vect }}
\end{array}\right]
$$

where $I_{3 \times 3}$ is the identity matrix and $\left[q_{r a, \text { vect }}^{\times}\right]$represents the skew-symmetric matrix defined as

$$
\left[q_{r a, \text { vect }}^{\times}\right]=\left[\begin{array}{c}
q_{r a, 1} \\
q_{r a, 2} \\
q_{r a, 3}
\end{array}\right]^{\times}=\left[\begin{array}{ccc}
0 & -q_{r a, 3} & q_{r a, 2} \\
q_{r a, 3} & 0 & -q_{r a, 1} \\
-q_{r a, 2} & q_{r a, 1} & 0
\end{array}\right] .
$$

More details about quaternion can be founded in [26].

$\bar{\omega}_{g}=\left[\begin{array}{ll}0 & \omega_{g}^{T}\end{array}\right]^{T}$ is the quaternion representation of the angular velocity $\omega_{g}=\left[\begin{array}{lll}\omega_{g x} & \omega_{g y} & \omega_{g z}\end{array}\right]^{T}$ expressed in $B$ [27], [28]. $\omega_{g}$ is measured by a three-axis gyroscope and can be often corrupted with noises and bias $\Lambda(t)$ [24], [29]. The operator $\otimes$ is defined in (3).

Equation (1) describes the time rate of attitude variation as a result of rigid body angular rates measured by the gyroscope. Including $\Lambda(t)$, (1) can be written as

$$
\dot{q}_{n}=\frac{1}{2}\left[\begin{array}{c}
-q_{n, \text { vect }}^{T} \\
I_{3 \times 3} q_{n, 0}+\left[q_{n, \text { vect }}^{\times}\right]
\end{array}\right]\left(\omega_{g}+\Lambda(t)\right)
$$

where $\left[q_{n \text {,vect }}^{\times}\right]$is defined in (3) and $I_{3 \times 3}$ is the identity matrix of dimension 3 .

The process model $(\Sigma)$ is composed of (5) and the output $y$

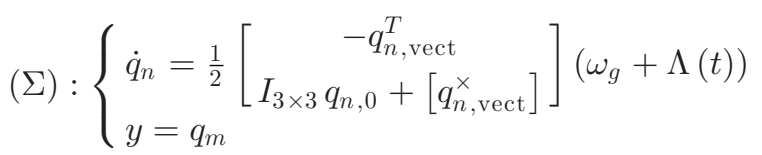

where $q_{n}$ is the mathematical representation of a rigid body real attitude expressed between two frames: 1) body-fixed frame $B$ and 2) Earth-fixed frame $N$. It represents the attitude variation derived from the rigid body angular rates integration. Acceleration and local magnetic field measurements are used to produce what is called the system's output $q_{m} \in \Re^{4 \times 1}$ based on the LMA.

\section{B. Inertial/Magnetic Sensors for Motion Estimation: $C O$}

$\mathrm{CO}$ design is described in Section II-A. The state vector is composed of four elements of quaternion. Fig. 1 shows the

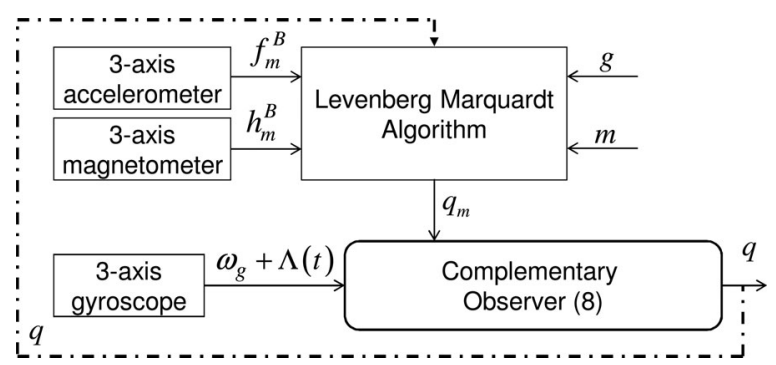

Fig. 1. Scheme of the estimation algorithm.

block diagram of the proposed approach. Acceleration and local magnetic field measurements are used as inputs to the LMA (see Section II-C) to produce the system's output. The system's output together with the angular rate measurements becomes inputs for the CO. Therefore, we can take advantages of the precision near to zero $\left(\prec 1^{\circ}\right)$, given by the rate gyros integration in the short term $(\approx 1 \mathrm{~s})$ and the reliable long-term accuracy provided by accelerometer / magnetometer [21].

The emphasis of the proposed observer is based on the multiplicative correction technique [26] written as follows:

$$
q(k+1)=\delta q(k+1) \otimes q(k)
$$

where $q(k+1)$ and $q(k)$ represent the post- and preupdate values of the quaternion estimates, respectively. Quaternion multiplication is used in (7) to correct and update the quaternion calculation. $\delta q(k+1)$ is the correction term which is a function of the error quaternion. This technique is more convenient for the transition between two quaternions in the case of large attitude motions. Finally, the CO can be designed from (5) and (7) as (8), shown at the bottom of the page, where $q$ is the estimated quaternion by the three sensors and $\left[q_{\text {vect }}^{\times}\right]$is defined in (4). $\delta_{K_{1}}$ represents the switching correction term and $\delta_{K_{2}}$ is the linear correction term. In order to perform the quaternion multiplication, each correction term should be converted into a quaternion. This conversion is obtained using the forced normalization method [30]. $\delta_{K_{1}}$ and $\delta_{K_{2}}$ are computed using

$$
\delta_{K_{1}}=\frac{1}{\left\|\chi_{1}\right\|} \chi_{1} ; \quad \delta_{K_{2}}=\frac{1}{\left\|\chi_{2}\right\|} \chi_{2}
$$

where

$$
\chi_{1}=K_{1}\left[\begin{array}{ll}
1 & \text { sat }
\end{array}\left[\frac{q_{e, \text { vect }}}{\rho}\right]\right]^{T} \quad \chi_{2}=K_{2}\left[\begin{array}{ll}
1 & q_{e, \text { vect }}
\end{array}\right]^{T}
$$

with

$$
K_{1}=\left[\begin{array}{cccc}
1 & 0 & 0 & 0 \\
0 & k_{1} & 0 & 0 \\
0 & 0 & k_{2} & 0 \\
0 & 0 & 0 & k_{3}
\end{array}\right] \quad K_{2}=\left[\begin{array}{cccc}
1 & 0 & 0 & 0 \\
0 & k_{4} & 0 & 0 \\
0 & 0 & k_{5} & 0 \\
0 & 0 & 0 & k_{6}
\end{array}\right]
$$

$$
\left\{\dot{q}=\left[\begin{array}{c}
\dot{q}_{0} \\
\dot{q}_{1} \\
\dot{q}_{2} \\
\dot{q}_{3}
\end{array}\right]=\delta_{K_{1}} \otimes \delta_{K_{2}} \otimes\left(\frac{1}{2}\left[\begin{array}{c}
-q_{\mathrm{vect}}^{T} \\
I_{3 \times 3} q_{0}+\left[q_{\mathrm{vect}}^{\times}\right]
\end{array}\right]\left(\left[\begin{array}{c}
\omega_{g x} \\
\omega_{g y} \\
\omega_{g z}
\end{array}\right]+\Lambda(t)\right)\right)\right.
$$


The term $q_{e, \text { vect }}$ in (10) represents the imaginary part of the error quaternion $q_{e} . q_{e}$ measures the difference between the complementary estimated quaternion $\bar{q}=$ $\left[\begin{array}{llll}q_{0} & -q_{1} & -q_{2} & -q_{3}\end{array}\right]^{T}$ and the system's output $q_{m} \cdot q_{e}$ is expressed by

$$
q_{e}=\bar{q} \otimes q_{m}=\left[\begin{array}{ll}
q_{e 0} & q_{e, \text { vect }}
\end{array}\right]^{T} .
$$

The scalar parts of $\chi_{1}$ and $\chi_{2}$ are chosen equal to unity since the incremental quaternion corresponds to a small rotation angle [26]. The saturation function in (10) is used to avoid the high-frequency chattering [31]. It can be written according to

$$
\text { sat }\left[q_{e, \text { vect }} / \rho\right]= \begin{cases}1, & q_{e, \text { vect }} \leq \rho \\ q_{e, \text { vect }} / \rho, & \left|q_{e, \text { vect }}\right| \leq|\rho| \\ -1, & q_{e, \text { vect }} \geq \rho .\end{cases}
$$

The parameter $\rho$ is the surface boundary layer. It determines the behavior in the vicinity of $q_{e, \text { vect }}=0$. To preserve the unit quaternion norm, the estimated quaternion $q$ in (8) can be normalized to avoid the divergence [30].

\section{System's Output $q_{m}$ Calculation: LMA}

The LMA is used for a single-frame estimation of the system's output $q_{m}$. The algorithm was proposed to solve the Wahba problem [32] that involved the determination of rigid body attitude in reference to a fixed coordinate system based on a set of observation vectors known in a fixed frame and measured in a mobile frame. A minimum of two vectors are required to compute the optimal attitude represented by $q_{m}$.

In the present case, the three-axis magnetometer is a sensor that provides the Earth's magnetic field $h$ in the body frame $B$. The theoretical output of a magnetometer can be written using the estimated quaternion such as [12]

$$
h=\left[\begin{array}{lll}
h_{x} & h_{y} & h_{z}
\end{array}\right]^{T}=M_{N}^{B}(q) m+\delta_{h}
$$

where $M_{N}^{B}(q)$ is the rotation matrix expressed as

$$
\begin{aligned}
& M_{N}^{B}(q)= \\
& {\left[\begin{array}{ccc}
2 q_{0}^{2}+2 q_{1}^{2}-1 & 2\left(q_{1} q_{2}+q_{0} q_{3}\right) & 2\left(q_{1} q_{3}-q_{0} q_{2}\right) \\
2\left(q_{1} q_{2}-q_{0} q_{3}\right) & 2 q_{0}^{2}+2 q_{2}^{2}-1 & 2\left(q_{0} q_{1}+q_{2} q_{3}\right) \\
2\left(q_{0} q_{2}+q_{1} q_{3}\right) & 2\left(q_{2} q_{3}-q_{0} q_{1}\right) & 2 q_{0}^{2}+2 q_{3}^{2}-1
\end{array}\right] .}
\end{aligned}
$$

$\delta_{h} \in \Re^{3}$ is a vector of white noise and $m$ represents the magnetic field vector measured in the Earth frame $N$ as

$$
m=\left[\begin{array}{lll}
m_{x} & 0 & m_{z}
\end{array}\right]^{T}=\left[\begin{array}{lll}
\|m\| \cos (\theta) & 0 & \|m\| \sin (\theta)
\end{array}\right]^{T} .
$$

The theoretical model of the magnetic field nearest to reality considers this vector with an inclination angle of $\theta=60^{\circ}$ and a vector norm of $\|m\|=0.5$ Gauss [33]. The magnetic field is locally constant in the fixed frame $N$ and can be represented by the vector $m$, which denotes the first observation vector.

The three-axis accelerometer measures the specific force $f$ in the body frame $B$ as follows [34]:

$$
f=\left[\begin{array}{lll}
f_{x} & f_{y} & f_{z}
\end{array}\right]^{T}=M_{N}^{B}(q)[a+g]+\delta_{f}
$$

where $g=\left[\begin{array}{lll}0 & 0 & 9.81\end{array}\right]^{T}$ is the gravity vector and $a=$ $\left[\begin{array}{lll}a_{x} & a_{y} & a_{z}\end{array}\right]$ is the linear acceleration of the body, expressed in the Earth frame $N$ [35], [36]. The rotation matrix $M_{N}^{B}(q)$ is expressed in (15) and $\delta_{f} \in \Re^{3}$ is a vector of white noise. In a quasi-static situation, the gravitational field $g$ dominates the accelerometer measurements $f\left(\|a\|_{2} \ll\|g\|_{2}\right)$ [21]. In this case, the quantity $g$ is also constant in the Earth frame $N$ and could provide the second observation vector.

The LMA is an estimator that uses the Earth's magnetic field $m$ and the gravity vector $g$ as the observation vectors and the real measurements $f_{m}^{B}$ and $h_{m}^{B}$ to deduce the system's output $q_{m}$. It is based on the following steps.

1) Measure the readings $f_{m}^{B}$ and $h_{m}^{B}$ from the accelerometer and magnetometer, respectively.

2) Calculate $\left[\begin{array}{ll}0 & f_{m}^{N}\end{array}\right]^{T}=q \otimes\left[\begin{array}{ll}0 & f_{m}^{B}\end{array}\right]^{T} \otimes \bar{q}$ and do the same for $\left[\begin{array}{ll}0 & h_{m}^{N}\end{array}\right]^{T} \cdot f_{m}^{N}$ and $h_{m}^{N}$ represent the estimated acceleration and magnetic field vectors in the Earth frame $N$.

3) Calculate the navigation errors $f_{e}=g-f_{m}^{N}$ and $h_{e}=$ $m-h_{m}^{N}$ in order to form $z=\left[\begin{array}{ll}h_{e} & f_{e}\end{array}\right]^{T}$.

4) Calculate the Jacobian matrix $J=-2$ $\left[\left[\left[h_{m}^{N}\right]^{\times}\right]^{T} \quad\left[\left[f_{m}^{N}\right]^{\times}\right]^{T}\right]^{T}$

5) Calculate the pseudoinverse $O^{*}=\left[J^{T} J+\lambda I_{3 \times 3}\right]^{-1} J^{T}$. The constant $\lambda$ is chosen small (between $10^{-1}$ and $10^{-2}$ ) to ensure the nonsingularity of the minimization problem [37].

6) Calculate the quaternion error such as $q_{\mathrm{er}}=\alpha O^{*} z$. $\alpha$ is a smooth parameter chosen between 0 and 1 [32].

7) Calculate $q_{m}$ such as $q_{m}=q \otimes\left[\begin{array}{ll}1 & q_{\mathrm{er}}\end{array}\right]^{T} . q$ is estimated at each integration step by the $\mathrm{CO}$.

\section{Performance Analysis of the Designed Observer}

A frequency analysis of inertial and magnetic sensor data shows that they have a complementary frequency spectrum [24]. The resulting structure of the proposed $\mathrm{CO}$ blends two frequency regions and is based on the complementary filtering theory [38].

1) The accelerometer and magnetometer data are characterized by a low-frequency region, where the attitude is usually more accurate.

2) The gyroscope data are located in the high-frequency region, where the integration of the angular velocity yields better attitude estimates.

To study the stability and performance of the $\mathrm{CO}$, we established the block diagram of the linearized quaternion observer (see Fig. 2). This block diagram results from the (6), (8)-(13), and the system's output $q_{m}$ issued from the LMA. We applied the Laplace transform ( $s$ is the Laplace operator) to $q_{m}, \dot{q}_{n}$, and $q$. The Laplace transformation (LT) of the quaternion $q_{m}$ is $q_{m}(s)$ and $s q_{n g}(s)$ is the LT of $\dot{q}_{n g}$. Here, we suppose that $\dot{q}_{n g}=\dot{q}_{n}$ where $q_{n g}$ is the quaternion obtained by integrating the three-axis gyroscope measurements using (6). In Fig. 2, the linearization led to approximate $\bar{q} \otimes q_{m}$ by $q_{m}-q$. More details on the linearization can be founded in [39]. 


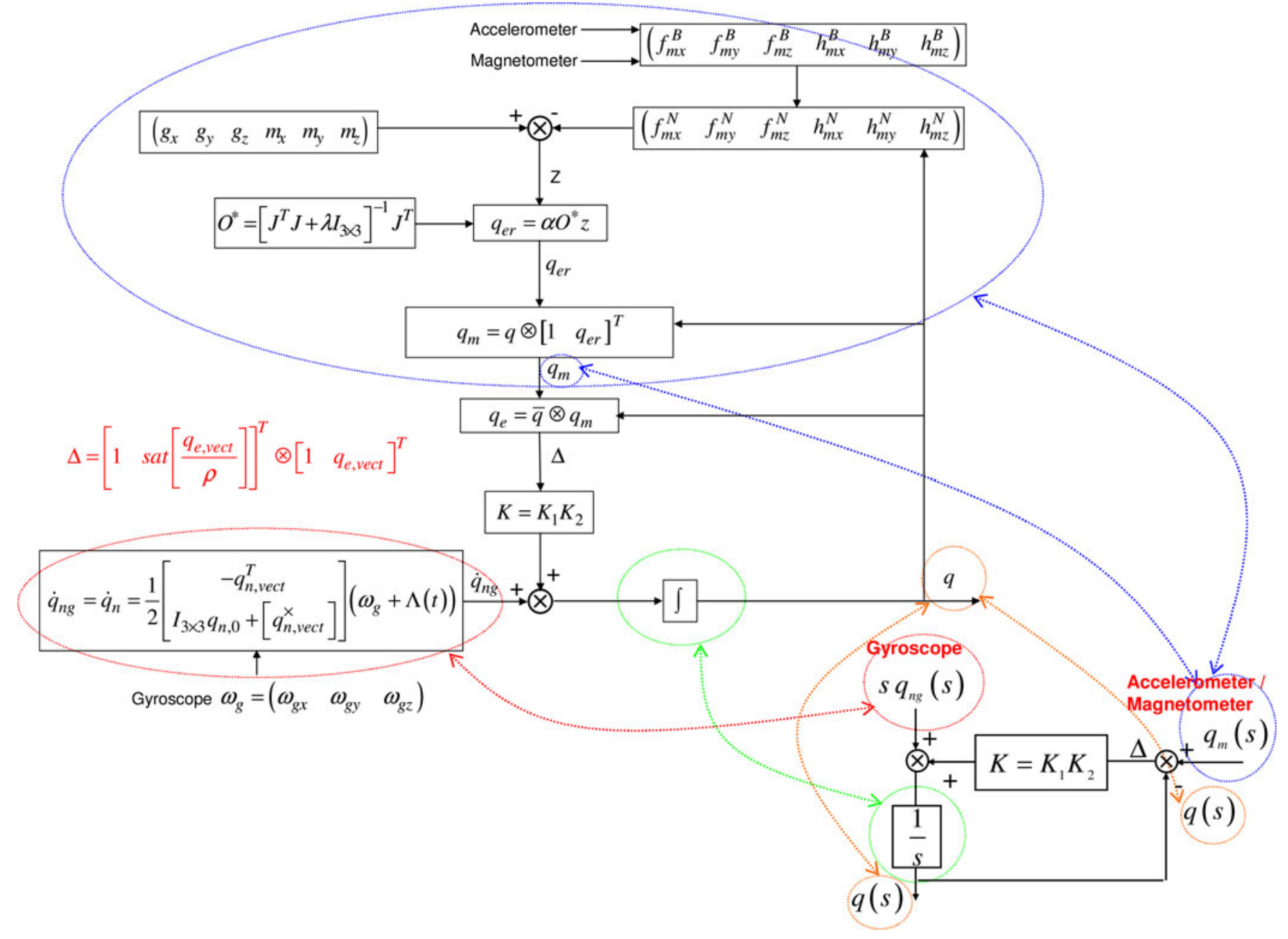

Fig. 2. Block diagram of the transform domain (Laplace) of the linearized quaternion observer.

From Fig. 2, the transfer function $F_{1}(s)$ based on the accelerometer and magnetometer inputs is given by

$$
F_{1}(s)=\frac{q(s)}{q_{m}(s)}=\frac{K s^{-1}}{1+K s^{-1}}=\frac{K}{s+K} .
$$

Equation (18) has the form of a first-order low-pass filter. Therefore, the system's output $q_{m}(s)$ is low-pass filtered with respect to $q(s)$. So, the perturbation effects due to the highfrequency components (resulting from the linear acceleration $a)$ of accelerometer signals are filtered from $q_{m}(s)$. The gain $K$ can be written as $K=K_{1} K_{2}$, where $K_{1}$ and $K_{2}$ are given in (11). Similarly, from Fig. 2, the transfer function based on the gyroscope inputs can be written as

$$
F_{2}(s)=\frac{q(s)}{q_{n g}(s)}=\frac{1}{1+K s^{-1}}=\frac{s}{s+K} .
$$

Equation (19) has the form of a first-order high-pass filter. The gyroscope measurements are high-pass filtered with respect to the output $q(s)$. Thus, the perturbations due to low-frequency components of gyroscope signal (the noises and biases) are filtered from $q_{g}(s)$.

The general requirement to prove the stability in the complementary filtering theory is that one of the transfer functions complements the sum of the others [22]. Then, this requirement can be verified for the $\mathrm{CO}$ by the following [22]:

$F_{1}(s)+F_{2}(s)=\frac{q(s)}{q_{m}(s)}+\frac{q(s)}{q_{n g}(s)}=\frac{K}{s+K}+\frac{s}{s+K}=1$.

The global transfer function of the $\mathrm{CO}$ is

$$
q(s)=\left(\frac{K}{s+K}\right) q_{m}(s)+\left(\frac{s}{s+K}\right) q_{n g}(s) .
$$

\section{EXPERIMENTAL METHODOLOGY}

\section{A. Experimental Tools for the Attitude Estimation: MTi and MTi-G}

In order to evaluate the efficiency of the proposed $\mathrm{CO}$ under several conditions in real applications, a set of experimental data was collected using two IMUs: the $M T i$ and the $M T i-G$. These sensor modules are designed by Xsens Technologies [40]. Sensing components for these units include a three-axis accelerometer, a three-axis magnetometer, and a three-axis gyroscope providing calibrated digital outputs at a rate of $100 \mathrm{~Hz}(3-\mathrm{D}$ acceleration, 3-D angular rate, and 3-D local magnetic field). The MTi- $G$ is a GPS-enhanced attitude and heading reference 


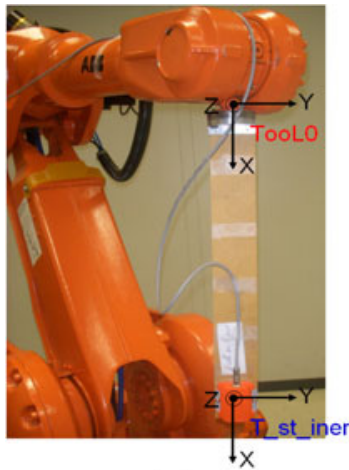

(a)

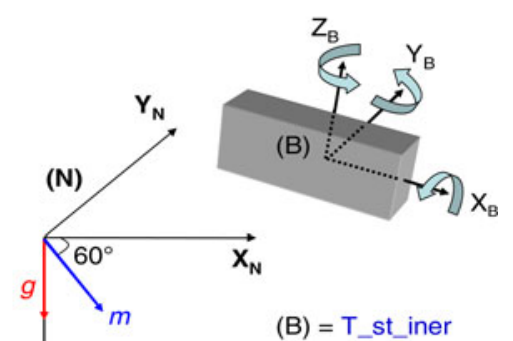

(b)
Fig. 3. (a) Experimental setup: the MTi is mounted on the robot for orientation tracking. (b) Coordinate system $(B)$ of a rigid body represented in the Earthfixed frame $(N)$.

system (AHRS). The calibration procedure of the sensors is performed by the manufacturer. The $M T i$ and the $M T i-G$ measure the attitude based on an embedded EKF [40]. The EKF combines measurements from the inertial/magnetic sensors and a GPS module (the GPS is only for the $M T i-G$ ) to estimate the attitude in quaternion or rotation matrix representations. In the following experiments, the calibrated data from the MTi and the $M T i-G$ are used as inputs to the CO.

\section{B. Experimental Setup}

The experimental setup used to evaluate the algorithm for the attitude estimation consists of three tests: 1) robot mounted tests; 2) improvement of the MTi shortcomings; and 3) human segments motion evaluation. The MATLAB computing program was used for all post-trial data processing and analysis.

1) Robot Mounted Tests: The experiments were carried out in the robotic laboratory of "PSA Peugeot Citroën" company based in Metz, France. The experimental setup used to evaluate the CO consists of an industrial robot IRB 2400 from the ABB Group [41] and the MTi. The robot is considered as the orientation reference since it offers an excellent motion control of around six axes and gives a high performance in the material handling with a position repeatability of $0.06 \mathrm{~mm}$ and $0.1^{\circ}$. The ranges of velocity rate used to move the robot are $[0-1 \mathrm{~m} / \mathrm{s}]$ and [0-20\%].

Fig. 3(a) shows the complete setup. In order to mitigate possible magnetic effects generated by the steel construction of the robot, the MTi is mounted on a nonferrous extension below the terminal effector of the robot. The extension is made of a piece of Plexiglas board and is approximately $50 \mathrm{~cm}$ in length. Two aligned frames are considered in this experimental setup: T_st_iner and TooLO correspond to the MTi and the terminal effector of robot, respectively [see Fig. 3(b)].

The reference paths for the six degrees of freedom of the IRB robot are generated with a programmed model (a trajectory like a straight line). The simulated motion is recorded and used afterward to move the IRB robot. The measured MTi signals are used for the estimation of the MTi's attitude with the proposed $\mathrm{CO}$. The estimated quaternion is compared to the one obtained

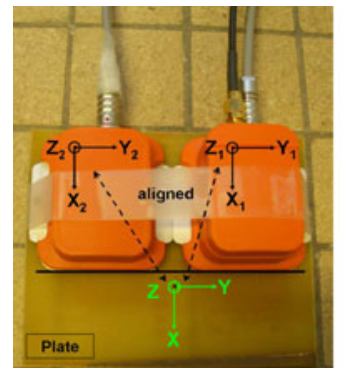

(a)

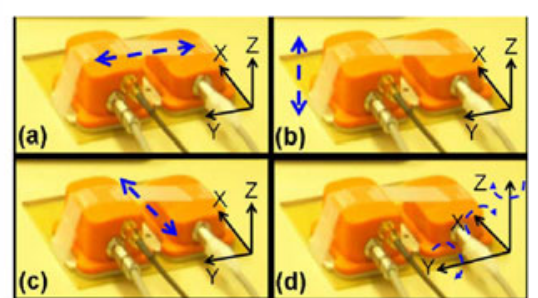

(b)
Fig. 4. (a) Whole system. The frame axes of a piece of Plexiglas board, MTi, and MTi-G are aligned (b) Motions performed during the evaluation test; the dashed arrow describes the direction of the motion.

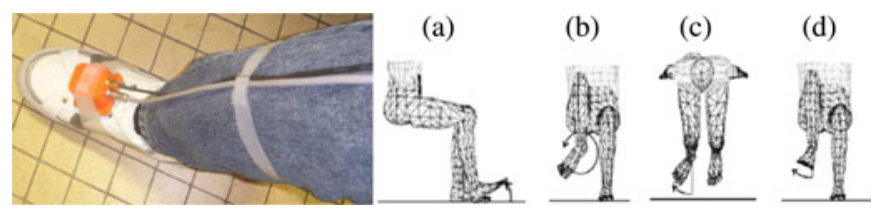

Fig. 5. Exercise 1 performed with the foot segment. (a) Toe rise foot. (b) Clockwise ankle rotation. (c) Lateral foot rotation. (d) Eversion.

from the incremental encoders of the IRB robot denoted as reference signals in the following. This motion is repeated four times by the robot to investigate the accuracy of the proposed filtering approach. During the experiment, we have chosen to increase the robot velocity at each test.

2) Improvement of the MTi Shortcomings: In this test, the goal is to elaborate an overall view of the attitude estimation using three methods: 1) the proposed $\mathrm{CO} ; 2$ ) the EKF in the $M T i$; and 3) the EKF in the MTi-G. The test procedure consists of attaching the MTi and the MTi-G to a piece of Plexiglas board using an adhesive strapping. Their frame axes are aligned as shown in Fig. 4(a). The subject took the overall package in his hand and is asked to perform the four motions outlined in Fig. 4(b) as follows: 1) move along the $y$-axis of the Plexiglas board in the level plane; 2) move along the $z$-axis of the Plexiglas board; 3 ) move along the $x$-axis of the Plexiglas board in the level plane; and 4) move around the $x$-axis, $y$-axis, and $z$-axis of the Plexiglas board (free motion).

3) Human Segments Motion Evaluation: Numerous experiments were conducted on a human subject to qualitatively evaluate the capability of the $\mathrm{CO}$ in the case of human body tracking. In each experiment, the $M T i-G$ is attached to the human limb to be tracked using an elastic strapping. In most cases, this method appeared to keep the sensors fixed relative to the limb. The MTi$G$ 's signals are used for an estimation of the limb's attitude with the proposed $\mathrm{CO}$. The estimated quaternion is compared to the one obtained from the MTi-G's EKF. To validate the effectiveness of the $\mathrm{CO}$, the experiments were chosen to cover a wide majority of the 3-D human motion. The subject was asked to perform four exercises on the following human segments: 1) the foot segment (see Fig. 5); 2) the lower leg segment (see Fig. 6); 3) the upper arm (see Fig. 7); and 4) the head (see Fig. 8).

In the first exercise, the subject performed the following four tasks with the foot segment for $80 \mathrm{~s}: 1$ ) a toe rise foot; 2) 


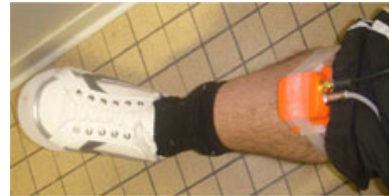

(a)

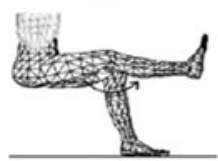

(b)

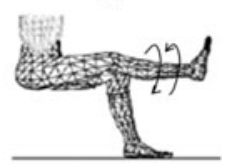

Fig. 6. Exercise 2 performed with the lower leg segment. (a) Knee extension. (b) Clockwise and anticlockwise rotation.

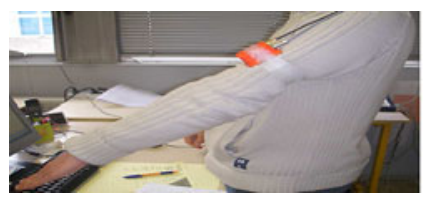

(a)

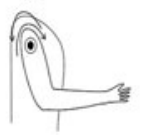

(b)

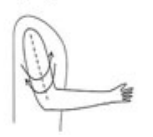

(c)

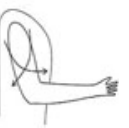

Fig. 7. Exercise 3 performed with the upper arm. (a) Clockwise and anticlockwise shoulder rotation. (b) Clockwise and anticlockwise rotation around the line defined along the upper arm segment. (c) Random motion.

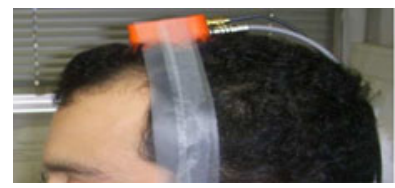

(a)

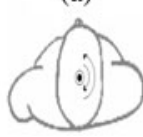

(b)

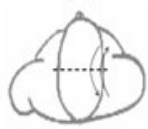

(c)

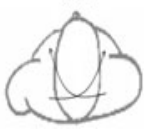

Fig. 8. Exercise 4 performed with the head. (a) Clockwise and anticlockwise neck rotation. (b) Clockwise and anticlockwise rotation around the dashed line lateral axis of the head. (c) Random motion.

a clockwise ankle rotation; 3) a lateral foot rotation; and 4) an eversion (see Fig. 5). In the second exercise, the subject carried out two tasks with the lower leg segment for $60 \mathrm{~s}: 1$ ) an extension of the knee and 2) a rotation of the leg in clockwise and anticlockwise directions (see Fig. 6). The third exercise is done on the upper arm for $60 \mathrm{~s}$. It consists of three tasks: 1) a shoulder rotation; 2) a rotation around the axis defined along the upper arm; and 3) a random motion (see Fig. 7). The exercises were completed by one performed on the head for $70 \mathrm{~s}$. The subject did three tasks: 1) a rotation of the neck in clockwise and anticlockwise directions; 2) a rotation around the lateral axis of the head; and 3) a random motion (see Fig. 8). To ensure legal and personal conditions, the experiments conformed to the National Center for Scientific Research (CNRS) Standard of Ethics.

\section{Results ANALYSIS AND Discussion}

\section{A. Robot Tests: Results and Performance Analysis}

Fig. 9(a) illustrates the evolution of the quaternion components in the case where the robot moves at $0.75 \mathrm{~m} / \mathrm{s}$. Fig. 9(a) obviously shows that the estimated quaternion by the $\mathrm{CO}$ is in good accordance with the reference signals calculated by the IRB robot. The observer is stable and estimates the truth attitude smoothly. Fig. 9(b) shows the quaternion estimation error between the $\mathrm{CO}$ and the reference (robot) after observer convergence. The obtained results illustrate that the quaternion estimation error remains in the interval that varies in \pm 0.015 which proves the efficiency of the $\mathrm{CO}$.

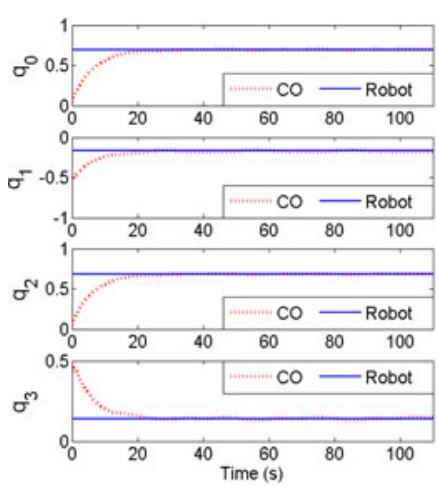

(a)

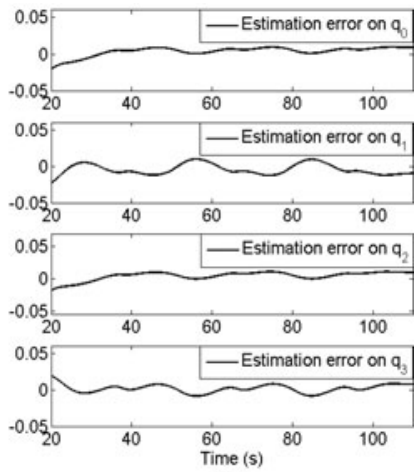

(b)
Fig. 9. (a) Comparison between quaternion components estimated by the $\mathrm{CO}$ and those given by the robot. (b) Quaternion estimation errors.

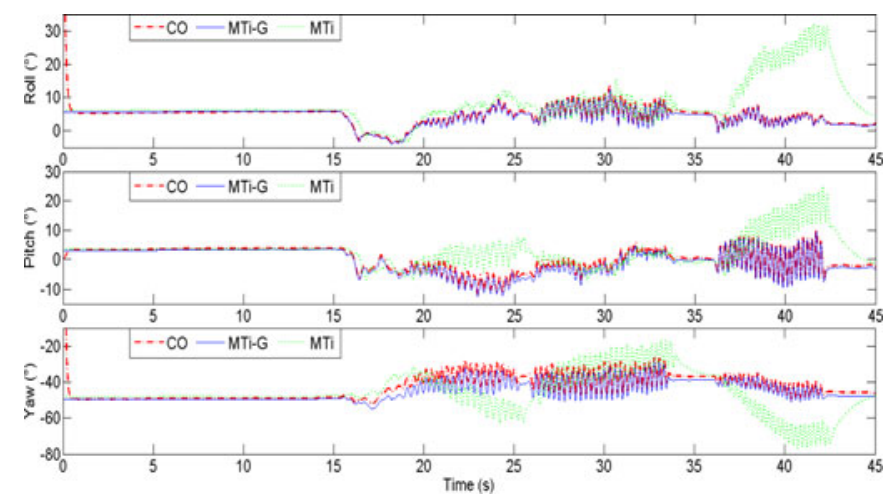

Fig. 10. Attitude estimation with the MTi, MTi-G, and CO.

\section{B. Improvement of the MTi Shortcomings: Results and Performance Analysis}

The $\mathrm{CO}$ is fed with inertial/magnetic data recorded from the $M T i-G$ during the four motions. The estimated attitude is expressed with the Euler angle representation. Fig. 10 shows the time history evolution of the Euler angles obtained from the MTi, the MTi-G, and the CO. This figure shows the effectiveness of the estimates produced by the $\mathrm{CO}$ during the motion. One can notice that the estimation error of quaternion remains close to zero (between $\left[1^{\circ}, 3^{\circ}\right]$ for roll and pitch angles and between $\left[2^{\circ}, 5^{\circ}\right]$ for a yaw angle). This experiment shows the drawbacks of the MTi's EKF during the intervals (17-34 s) and (37-42 s) where the estimation errors are between $10^{\circ}$ and $20^{\circ}$.

\section{Human Segments Motion Evaluation: Results and Performance Analysis}

The estimated attitude by the CO is compared to the one obtained from the $M T i-G$ 's EKF considered as reference in each exercise. The attitude is expressed also in the Euler angle representation using the mathematical transformation between the estimated quaternion and Euler angles given in [42]. Fig. 11(a) illustrates the obtained results from the exercise on the upper arm as an illustrative example. The time history evolution of the Euler angles obtained from the MTi-G's EKF and the CO is shown in this figure. We obviously deduce a strong correlation between the attitudes obtained from the EKF and the $\mathrm{CO}$, 

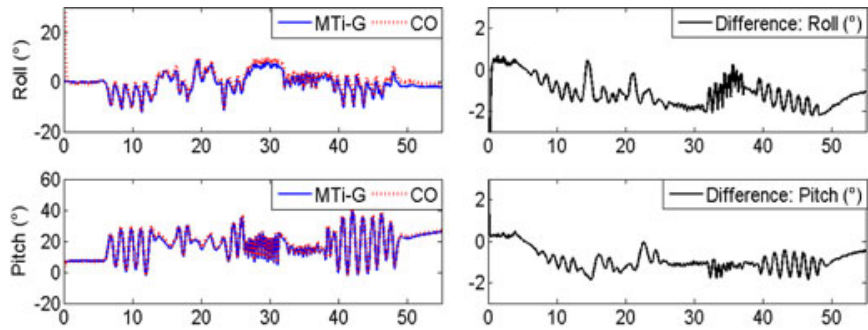

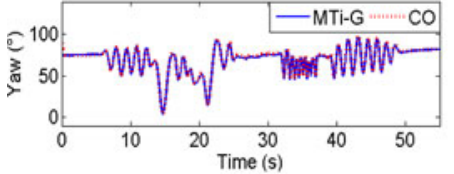

(a)

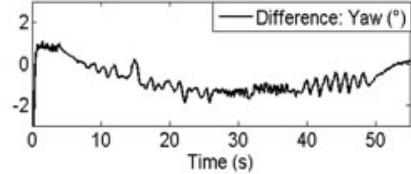

(b)
Fig. 11. (a) Euler angles estimated by the $\mathrm{CO}$ and those obtained by the MTi-G's EKF. (b) Estimation errors (exercise on the upper arm).

respectively. The convergence rate is fast and around $2 \mathrm{~s}$. The Euler angle estimation errors are shown in Fig. 11(b), which provide an overview of the overall performance of the proposed approach in this paper. These errors are computed as the difference between the Euler angle estimates produced by the $\mathrm{CO}$ and the EKF, respectively. The performance consistency of the observer is illustrated in this figure since the estimation errors are around $2^{\circ}$ for the roll and pitch angles and $3^{\circ}$ for the yaw angle. This test shows the attitude estimation capability of the $\mathrm{CO}$ without resorting to the GPS data (GPS data are used only in the $M T i-G$ 's EKF). During this test, the observer was evaluated under a large range of movement and the estimation errors were very similar (between $2^{\circ}$ and $4^{\circ}$ for the roll and pitch angles, between $3^{\circ}$ and $5^{\circ}$ for the yaw angle).

\section{CONCLUSION}

This paper has presented the design and experimental results of a quaternion-based $\mathrm{CO}$ for human body motion tracking using inertial/magnetic sensor modules containing orthogonally mounted triads of accelerometers, gyroscopes, and magnetometers. The $\mathrm{CO}$ was designed with the goal of being able to produce highly accurate orientation estimates without resorting to GPS data. The observer design makes use of a simple kinematic motion equation to describe the system model. The CO design is further simplified by preprocessing accelerometer and magnetometer data using the LMA. The quaternion calculated by the LMA is provided as input to the $\mathrm{CO}$ along with the angular rate data. Some experiments were carried out on a robot and human body segments through sensor measurements provided by an IMU. The obtained results and estimation errors illustrate the performance of the proposed approach. Future work will focus on designing a low-cost, lightweight, and embedded prototype for this application.

\section{REFERENCES}

[1] H. Zhou, H. Hu, N. D. Harris, and J. Hammerton, "Applications of wearable inertial sensors in estimation of upper limb movements," Biomed. Signal Process. Control, vol. 1, no. 1, pp. 22-32, Jan. 2006.
[2] A. M. Sabatini, "Quaternion-based strap-down integration method for applications of inertial sensing to gait analysis," Med. Biol. Eng. Comput., vol. 43, no. 1, pp. 94-101, Jan. 2005.

[3] H. Zhou and H. Hu, "Human motion tracking for rehabilitation-A survey," Biomed. Signal Process. Control, vol. 3, no. 1, pp. 1-18, Jan. 2008.

[4] P. H. Veltink, H. Kortier, and H. Martin Schepers, "Sensing power transfer between the human body and the environment," IEEE Trans. Biomed. Eng., vol. 56, no. 6, pp. 1711-1718, Jun. 2009.

[5] A. U. Alahakone and S. M. N. Arosha Senanayake, "A real-time system with assistive feedback for postural control in rehabilitation," IEEE/ASME Trans. Mechatronics, vol. 15, no. 2, pp. 226-233, Apr. 2010.

[6] Gypsy4 Mechanical Motion Capture. (2004). [Online]. Available: www.animazoo.com

[7] Motion Capture Systems From Vicon. (2005). [Online]. Available: www.vicon.com

[8] S. Beeby, G. Ensell, M. Kraft, and N. White, MEMS Mechanical Sensors. Norwood, MA: Artech House, 2004.

[9] C. Verplaeste, "Inertial proprioceptive devices: Self-motion-sensing toys and tools," IBM Syst. J., vol. 35, no. 3-4, pp. 639-650, 1996.

[10] H. J. Luinge and P. H. Veltink, "Inclination measurement of human movement using a 3-D accelerometer with autocalibration," IEEE Trans. Neural Syst. Rehabil. Eng., vol. 12, no. 1, pp. 112-121, Mar. 2004.

[11] J. E. Bortz, "A new mathematical formulation for strap-down inertial navigation," IEEE Trans. Aerosp. Electron. Syst., vol. 7, no. 1, pp. 61-66, Jan. 1971.

[12] M. J. Caruso, "Applications of magnetic sensors for low cost compass systems," in Proc. IEEE Position, Location Navig. Symp., San Diego, CA, 2000, pp. 177-184.

[13] Eric R. Bachmann, X. Yun, and A. Brumfield, "Limitations of attitude estimation algorithms for inertial/magnetic sensor modules," IEEE Robot. Autom. Mag., vol. 14, no. 3, pp. 76-87, Sep. 2007.

[14] H. J. Luinge and P. H. Veltink, "Measuring orientation of human body segments using miniature gyroscopes and accelerometers," Med. Biol. Eng. Comput., vol. 43, no. 2, pp. 273-282, 2005.

[15] R. Zhu and Z. Zhou, "A real-time articulated human motion tracking using tri-axis inertial/magnetic sensors package," IEEE Trans. Neural Syst. Rehabil. Eng., vol. 12, no. 2, pp. 295-302, Jun. 2004.

[16] X. Yun and Eric R. Bachmann, "Design, implementation, and experimental results of a quaternion-based Kalman filter for human body motion tracking," IEEE Trans. Robot., vol. 22, no. 6, pp. 1216-1227, Dec. 2006.

[17] H. Ren and P. Kazanzides, "Investigation of attitude tracking using an integrated inertial and magnetic navigation system for hand-held surgical instruments," IEEE/ASME Trans. Mechatronics, vol. 17, no. 2, pp. 210217, Apr. 2012

[18] K. D. Nguyen, I.-M. Chen, Z. Luo, S. H. Yeo, and H. B.-L. Duh, "A wearable sensing system for tracking and monitoring of functional arm movement," IEEE/ASME Trans. Mechatronics, vol. 16, no. 2, pp. 213220, Apr. 2011.

[19] Z. Q. Zhang, X. L. Meng, and J. K. Wu, "Quaternion-based Kalman filter with vector selection for accurate orientation tracking," IEEE Trans. Instrum. Meas., vol. 61, no. 10, pp. 2817-2824, Oct. 2012.

[20] A. A. Gopalai and S. M. N. A. Senanayake, "A wearable real-time intelligent posture corrective system using vibrotactile feedback," IEEE/ASME Trans. Mechatronics, vol. 16, no. 5, pp. 827-834, Oct. 2011.

[21] R. Mahony, T. Hamel, and J. M. Pflimlin, "Nonlinear complementary filters on the special orthogonal group," IEEE Trans. Autom. Control., vol. 53, no. 5, pp. 1203-1218, Jun. 2008.

[22] H. Fourati, N. Manamanni, L. Afilal, and Y. Handrich, "Nonlinear filtering approach for the attitude and dynamic body acceleration estimation based on inertial and magnetic sensors: Bio-logging application," IEEE Sensors J., vol. 11, no. 1, pp. 233-244, Jan. 2011.

[23] H. Grip, T. I. Fossen, T. A. Johansen, and A. Saberi, "Attitude estimation using biased gyro and vector measurements with time-varying reference vectors," IEEE Trans. Autom. Control, vol. 57, no. 5, pp. 1332-1338, May 2012.

[24] R. G. Brown and P. Y. C. Hwang, Introduction to Random Signal and Applied Kalman Filtering, 3rd ed. New York: Wiley, 1997.

[25] M. D. Shuster, "A survey of attitude representations," J. Astronaut. Sci., vol. 41, no. 4, pp. 493-517, Oct./Dec. 1993.

[26] J. B. Kuipers, Quaternion and Rotation Sequences. Princeton, NJ: Princeton Univ. Press, 1999.

[27] P. Cardou, G. Fournier, and P. Gagnon, "A nonlinear program for angular velocity estimation from centripetal acceleration measurements," IEEE/ASME Trans. Mechatronics, vol. 16, no. 5, pp. 932-944, Oct. 2011. 
[28] J. K. Lee, E. J. Park, and S. N. Robinovitch, "Estimation of attitude and external acceleration using inertial sensor measurement during various dynamic conditions," IEEE Trans. Instrum. Meas., vol. 61, no. 8, pp. 22622273, Aug. 2012.

[29] H. Fourati, N. Manamanni, L. Afilal, and Y. Handrich, "Posture and body acceleration tracking by inertial and magnetic sensing: Application in behavioral analysis of free-ranging animals," Biomed. Signal Process. Control, vol. 6, no. 1, pp. 94-104, Jan. 2011.

[30] J. Deutschmann, I. Bar-Itzhack, and K. Galal, "Quaternion normalization in spacecraft attitude determination," in Proc. AIAA Astrodynamics Conf., Washington, DC, 1992, pp. 27-37.

[31] J. J. E. Slotine, J. K. Hedrick, and A. Misawa, "On sliding observers for nonlinear systems," ASME J. Dyn. Syst. Meas. Control, vol. 109, pp. 245252, 1987.

[32] G. Wahba, "A least squares estimate of spacecraft attitude," SIAM Rev., vol. 7, no. 3, p. 409, Jul. 1965.

[33] Astrosurf. (2011, Jun.). http://www.astrosurf.com

[34] P. Lin, J. Lu, C. Tsai, and C. Ho, "Design and implementation of a nineaxis inertial measurement unit," IEEE/ASME Trans. Mechatronics, vol. 17, no. 4, pp. 657-668, Apr. 2012.

[35] W. Wang and O. A. Jianu, "A smart sensing unit for vibration measurement and monitoring," IEEE/ASME Trans. Mechatronics, vol. 15, no. 1, pp. 7078, Feb. 2010

[36] H. Fourati, N. Manamanni, L. Afilal, and Y. Handrich, "A quaternionbased complementary sliding mode observer for attitude estimation: application in free-ranging animal motions," in Proc. 49th IEEE Conf. Decision Control, Atlanta, GA, 2010, pp. 5056-5061.

[37] J. E. Dennis, Jr. and B. Robert Schnabel, Numerical Methods for Unconstrained Optimization and Nonlinear Equations. Englewood, NJ: Prentice-Hall, 1983.

[38] W. Higgins, "A comparison of complementary and Kalman filtering," IEEE Trans. Aerosp. Electron. Syst., vol. 11, no. 3, pp. 321-325, May 1975.

[39] R. B. McGhee, E. R. Bachmann, X. Yun, and M. J. Zyda, "Real-time tracking and display of human limb segment motions using sourceless sensors and a quaternion based filtering algorithm-Part I: Theory," Naval Postgraduate School, Monterey, CA, Rep. Doc., Nov. 2000.

[40] Xsens Technologies. (2012, Oct.). http://www.xsens.com

[41] ABB Group. (2012, Oct.). http://www.abb.com

[42] W. F. Phillips, C. E. Hailey, and G. A. Gebert, "Review of attitude representations used for aircraft kinematics," J. Aircraft, vol. 38, no. 4, pp. 718223, Jul./Aug. 2001.

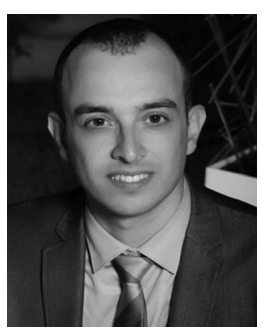

Hassen Fourati received the B.Eng. degree in electrical engineering from the National Engineering School of Sfax, Sfax, Tunisia, the M.A. degree in automated systems and control from the University of Claude Bernard, Lyon, France, and the Ph.D. degree in automatic control from the University of Strasbourg, Strasbourg, France, in 2006, 2007, and 2010, respectively.

$\mathrm{He}$ is currently an Assistant Professor at the University of Joseph Fourier, Grenoble, France, and a member of the Networked Controlled Systems Team (NeCS), affiliated with the GIPSA-LAB laboratory. His research interests include nonlinear filtering, estimation and multisensor fusion with applications in robotics, biologging, and road traffic.

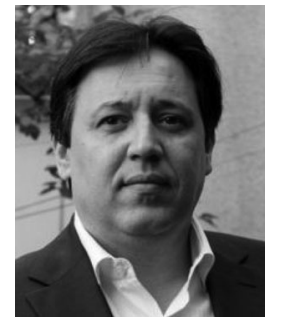

Noureddine Manamanni (M'07) received the M.Eng. degree in electrical engineering from the University of Annaba, Algeria, in 1990. After two years of experience in industry he received the Ph.D. degree in robotics and automation from the Universite Pierre et Marie Curie (UPMC) Paris VI, Paris, France, in 1998.

He is currently a Full Professor with the University of Reims Champagne-Ardenne, Reims, France, and is affiliated with the CReSTIC-Lab, Reims. $\mathrm{He}$ is heading the Automatic Control and Hybrid Systems Research Group, CReSTIC. His research interests include nonlinear observer design, estimation, nonlinear control, Lyapunov stability, hybrid dynamical systems, control of robotic systems, and their application.

Prof. Manamanni is a member of three IFAC Technical Committees and has been an IEEE member since 2007 in five Societies, among them Control Systems (CSS), CIS, ITSS, and RAS.

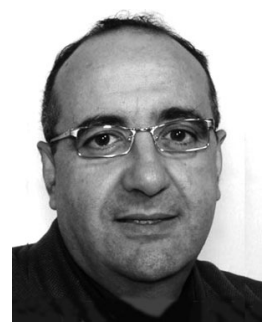

Lissan Afilal received the "Matrise de Physique" degree and the Ph.D. degree in automatic control and computer science from the University of Reims Champagne-Ardenne (URCA), in 1984 and 1990, respectively.

$\mathrm{He}$ runs the theme of embedded systems in the Automatic Control and Hybrid System Research Group, CReSTIC Centre de Recherche en Sciences et Technologies de l'Information et de la Communication, Reims, France. His research interests include automatic biomedical engineering, the control of complex systems, computer engineering, and the design of embedded systems. His application areas range from the interoperability of systems on vehicles to bio-logging.

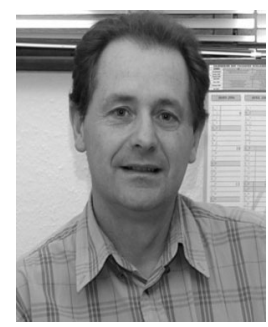

Yves Handrich was born in Paris, France, in 1959. He received the "Aggregation de physiology animale" in 1984 and the Ph.D. degree in animal ecophysiology from the University of Louis Pasteur, Strasbourg, France, in 1989.

In 1989, he became a Researcher with the "Centre National de la Recherche Scientific (CNRS)," Strasbourg, France. Since 1989, he has been with CNRS (which became the "Institut Pluridisciplinaire Hubert Curien" (IPHC-CNRS) in 2007), Strasbourg, France. His research field is focused on the behavioral energetic and ecophysiology of wild animal living in extreme environmental conditions. He is a specialist on fasting, thermoregulation, and diving in marine birds One key of his approach is the use of the bio-logging technology. 